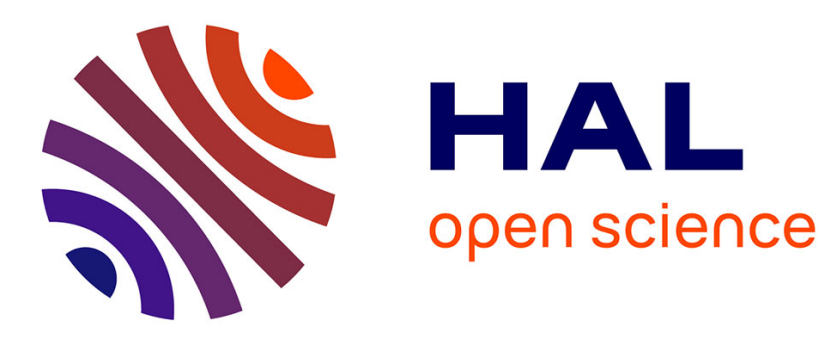

\title{
Camera tracking by online learning of keypoint arrangements using LLAH in augmented reality applications
}

Hideaki Uchiyama, Hideo Saito, Myriam Servières, Guillaume Moreau

\section{To cite this version:}

Hideaki Uchiyama, Hideo Saito, Myriam Servières, Guillaume Moreau. Camera tracking by online learning of keypoint arrangements using LLAH in augmented reality applications. Virtual Reality, 2011, 15, 10.1007/s10055-010-0173-7 . hal-01521143

HAL Id: hal-01521143

https://hal.science/hal-01521143

Submitted on 11 May 2017

HAL is a multi-disciplinary open access archive for the deposit and dissemination of scientific research documents, whether they are published or not. The documents may come from teaching and research institutions in France or abroad, or from public or private research centers.
L'archive ouverte pluridisciplinaire HAL, est destinée au dépôt et à la diffusion de documents scientifiques de niveau recherche, publiés ou non, émanant des établissements d'enseignement et de recherche français ou étrangers, des laboratoires publics ou privés. 


\title{
Camera tracking by online learning of keypoint arrangements using LLAH for augmented reality applications
}

\author{
Hideaki Uchiyama · Hideo Saito . \\ Myriam Servières · Guillaume Moreau
}

Received: date / Accepted: date

\begin{abstract}
We propose a camera tracking method by on-line learning of keypoint arrangements for augmented reality applications. As target objects, we deal with intersection maps from GIS and text documents, which are cannot be handled by the popular SIFT and SURF descriptors. For keypoint matching by keypoint arrangement, we use locally likely arrangement hashing (LLAH), in which the descriptors of the arrangement are not invariant to wide viewpoints because local arrangement is changeable with respect to viewpoints. In order to solve this problem, we propose online learning of descriptors using new configurations of keypoints at new viewpoints. The proposed method allows keypoint matching to proceed under new viewpoints. We evaluate the performance and robustness of our tracking method using view changes.
\end{abstract}

Keywords LLAH · Feature Descriptor · Camera Tracking · Augmented Reality

\section{Introduction}

Camera tracking remains an open and active fundamental problem in the computer vision domain. In particular, augmented reality (AR) applications need real-time processing and robust camera tracking in order to place virtual objects in an actual scene. To meet those requirements, several approaches have been proposed.

Fiducial markers have been widely developed for a long time, and they have been used in many AR frameworks (Kato and Billinghurst, 1999; Fiala, 2005; Wagner et al, 2008a). In fact, these marker systems are already used in practical applications in companies and industries (Pentenrieder et al, 2007).

Recently, the focus of research has been shifting towards the use of natural features from actual environments such as edges and feature points, because fiducial markers may not be

Hideaki Uchiyama $\cdot$ Hideo Saito

Keio University, 3-14-1 Hiyoshi, Kohoku-ku 223-8522, Japan

E-mail: \{uchiyama,saito\}@hvrl.ics.keio.ac.jp

Myriam Servières · Guillaume Moreau

Ecole Centrale de Nantes - CERMA IRSTV, Rue de la Noë, BP 92101, 44321 Nantes Cedex 3, France

E-mail: \{myriam.servieres, guillaume.moreau\}@ec-nantes.fr 
available (for instance, because of installation permission in outdoor environments, sizebased issues, and application constraints). For example, edge-based approaches can be found in model-based tracking (Drummond and Cipolla, 1999), initialization of tracking (Kotake et al, 2007) and visual SLAM (Klein and Murray, 2008) using boundaries of a room and the rims of a non textured object. The keypoint (feature point) matching based approach is also becoming common, owing to the development of local descriptors such as SIFT (Lowe, 2004). In addition, the computational complexity of this approach is drastically decreasing and allows for the implementation of the method on a mobile device with a low speed CPU and less memory, as in Phony SIFT (Wagner et al, 2008b).

Even though this remarkable development has already been achieved, this approach cannot be applied to our target objects such as maps that include only intersections as simple circular dots (Uchiyama et al, 2008, 2009) and text documents having locally repetitive patterns (Uchiyama and Saito, 2009), because rich textured objects are necessary for the descriptors. Instead of the local patch based descriptors, the local arrangement of keypoints is shown as a distinctive descriptor in such cases.

In this study, we describe a camera tracking method for our target objects, which cannot be tracked by traditional methods. We use LLAH (Nakai et al, 2005) to describe the local arrangement of keypoints. Because the local arrangement might be modified when the viewpoint changes, we propose a method for learning the new configuration of keypoints at new viewpoints, in order to handle a large range of viewpoint changes.

The rest of this paper is arranged as follows. The next section describes related studies regarding keypoint matching. In particular, the details of LLAH are highlighted in Section 3, because LLAH is an important component in our method. Section 4 explains our main contribution to the learning process with LLAH. Section 5 demonstrates the performance and robustness of our method. Section 6 discusses our conclusions and future work.

\section{Related works}

The entire process of keypoint matching can be divided into three parts: extraction, description and matching.

For keypoint extraction, Harris corner (Harris and Stephens, 1988) and FAST corner (Rosten and Drummond, 2006) have been proposed for extracting keypoints that have a different appearance from their neighboring pixels. These methods can be applied to multiscale images to take into account scale changes. There have been several approaches for extracting scale-invariant feature points, such as the difference of Gaussians (Lowe, 2004), gradient locations and orientation histograms (Mikolajczyk and Schmid, 2005), and basic Hessianmatrix approximation (Bay et al, 2008).

A keypoint descriptor is a high dimensional vector computed from the local neighbor region of the keypoint in order to construct a discriminative power. Descriptors such as SIFT (Lowe, 2004) and SURF (Bay et al, 2008) have been designed to be invariant to illumination, scale, rotation, and translation changes. Because they require high computational power, several attempts have been made to accelerate the computation of such descriptors. In particular, it is important to run at interactive frame rates in order to provide real-time applications and interaction with the user in AR systems. Among them, Sinha (Sinha et al, 2006) has implemented SIFT on a GPU in order to use parallel processing. Wagner (Wagner et al, 2008b) has proposed Phony SIFT, which is a mobile phone version of SIFT that removes some computational costs related to keypoint extraction and descriptor computation. 
The matching of descriptors can be addressed as a nearest neighbor searching problem between high dimensional vectors. Approximate nearest neighbor is a searching method based on kd-trees and box-decomposition trees (Arya et al, 1998). Because a distance computation is performed for the comparison between two vectors, the retrieval cost depends on the dimension of the vector. Locality sensitive hashing (LSH) is another approximate searching method based on probabilistic dimension reduction with a hash scheme (Datar et al, 2004). The computational cost of LSH is always $O(1)$, but the nearest neighbor points might not be found. The design of the hash function remains an important issue in order to efficiently store data, which requires having as few collisions as possible in the hash table. Nister and Stewenius have proposed a recursive k-means tree as a vocabulary tree for quick retrieval (Nister and Stewenius, 2006). Lepetit et al. have treated the matching of descriptors as a classification problem (Lepetit et al, 2004).

Local descriptors are well suited to match keypoints with rich texture patterns. In contrast, these descriptors cannot be applied to our targets, such as intersection maps and text documents. For example, the local textures in the intersection maps are the same because the texture is only composed of identical circular dots. In this case, descriptors such as SIFT and SURF do not work well because local areas do not have enough discriminative power to be distinct from other areas. In addition, in text documents, local textures are almost identical and cannot be described by SIFT and SURF. Instead of local patch based descriptors, we promote the use of descriptors that consider the geometrical relationship between keypoints, which has already been proposed in studies of document image retrieval (Hull et al, 2007; Nakai et al, 2005).

Hull et al. have proposed to use the horizontal connectivity of word lengths as a descriptor (Hull et al, 2007). Word length refers to the number of characters and is linked with the previous and next word lengths. Because word lengths are very sensitive to viewpoint changes, this descriptor is valid only when a user captures an image where the camera is orthogonal to the document and close enough to the paper. Text lines must also be parallel to the lower side of the image.

Nakai et al. have proposed keypoint matching using the local arrangement of keypoints for document image retrieval, called LLAH (Nakai et al, 2005). The objective is to quickly find a document relevant to a query image from a database containing numerous documents. LLAH is an improved method of geometric hashing (GH) (Lamdan and Wolfson, 1988) in terms of memory use and computational cost. Because the computational cost of GH considering perspective distortion is $O\left(N^{5}\right)$, where $N$ is the number of keypoints in a query, it is difficult to apply it to real-time applications, such as AR systems. To solve the computational cost problem, LLAH focuses only on local geometry with neighbor keypoints. However, the descriptors of local arrangement in LLAH are invariant within a narrow view because the arrangement is changeable with respect to viewpoints. In order to handle a large range of viewpoints, we merge the online learning of the new configuration of keypoints into LLAH.

\section{Descriptors in LLAH}

In this section, we explain the descriptors in LLAH because our method is mainly based on these descriptors (Nakai et al, 2006).

In Figure 1, $\mathbf{x}$ is an example target keypoint. First, the $n$ nearest neighbor points around $\mathbf{x}$ are selected as abcdefg $(n=7)$. The order to select the $n$ points should be defined beforehand. For example, we select from $\mathbf{a}$ in a counterclockwise fashion based on the reference axes, as illustrated in Figure 1. 
Next, $m$ points out of $n$ points are selected as abcde $(m=5)$. From these $m$ points, one descriptor is computed. Because a descriptor is computed on each combination, a keypoint has ${ }_{n} C_{m}=\frac{n !}{m !(n-m) !}$ descriptors.

From $m$ points, $l$ points are selected for computing a geometrical invariant. As the invariant, Nakai et al. selected a cross ratio as a perspective invariant (Nakai et al, 2005) and a ratio of two triangles as an affine invariant (Nakai et al, 2006). Because they concluded that the affine invariant was better (because the perspective invariant was not stably computed), we select the affine invariant $(l=4)$.

In Figure 1, four points are selected as abcd to compute a ratio of two triangles. For a hashing scheme, the value of the ratio is quantized into an index using a distribution histogram created in prior experiments (Nakai et al, 2005). The histogram is segmented into the number of quantization level to assign an integer number at each segment. Because the number of the combination to select four points is equivalent to the dimension of the descriptor, the dimension is ${ }_{m} C_{4}$.

For quick retrieval, a hash scheme is adopted. The descriptor is converted into an index using following equation:

$$
\text { Index }=\left(\sum_{i=0}^{m C_{l}-1} r_{(i)} k^{i}\right) \bmod H_{\text {size }}
$$

where $r_{(i)}\left(i=0,1, \ldots, m C_{l}-1\right)$ are quantized values of geometrical invariants, $k$ is the quantization level and $H_{\text {size }}$ is the pre-defined hash size. As a result, each keypoint is stored in a hash table as (Index, Document ID + Keypoint ID). The table has been pre-defined to a large size to access the element of each index by $O(1)$. In addition, a keypoint database is prepared to store a 2D coordinate of each keypoint as (Document ID + Keypoint ID, 2D coordinate). In our implementation, a document ID and a keypoint ID are represented by a 32 bit ID by assigning 16 bits to both IDs.

When a keypoint is stored in the hash table, a collision occurs at the index. Because the discriminative power of such an index is considered to be too low, a keypoint is not stored at the index.

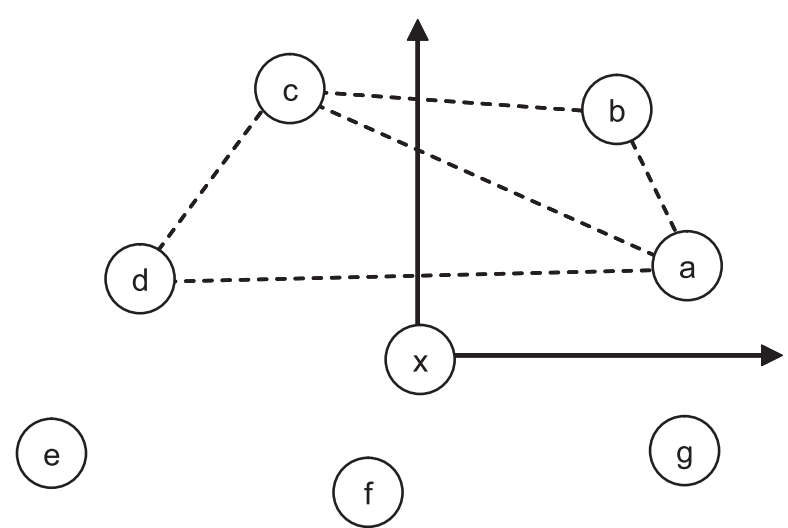

Fig. 1 Descriptors in LLAH. The descriptors of a keypoint are computed from the combination of the ratio of two triangles. 


\section{Proposed method}

\subsection{Target objects and their keypoints}

Our target objects for augmented reality applications are intersection maps (Uchiyama et al, 2008, 2009) and text documents (Uchiyama and Saito, 2009). Figure 2(a) is an example of intersection maps generated from a Geographical Information System (GIS), and Figure 2(b) is the visualization of 3D data on GIS. This application was developed to achieve a novel geo-visualization by relating GIS and paper maps. Also, Figure 2(c) illustrates a virtual annotation system implemented as augmented reality on a document.

In these applications, local patch based tracking methods do not work because local texture patterns are not sufficiently distinctive. For this reason, we sought another approach using the local arrangement of keypoints as a descriptor.

For keypoint extraction, the intersections are extracted by color extraction because the intersections have a specific color, such as red. From text documents, word regions are extracted using adaptive thresholding in the same way as (Nakai et al, 2006). A keypoint is the center of each region. Compared to normal textures, local texture patterns are similar, but keypoints are stably extracted.

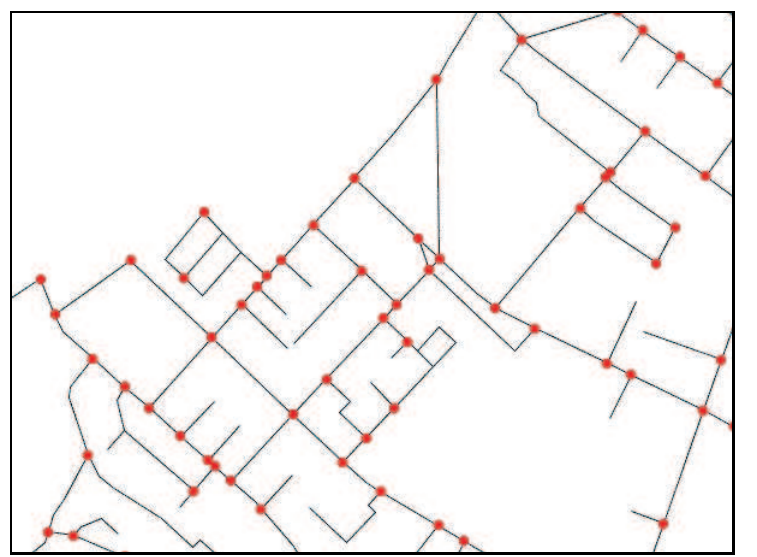

(a)

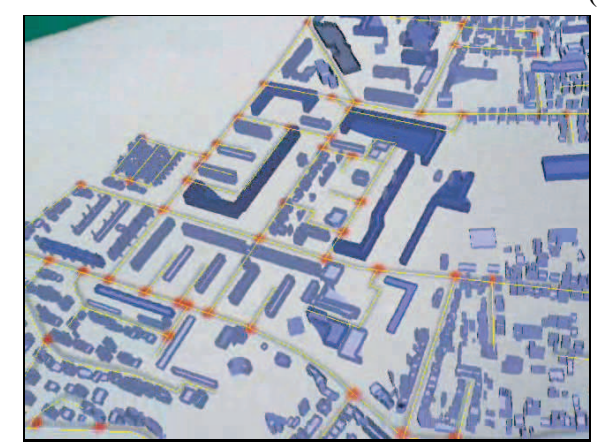

(b)

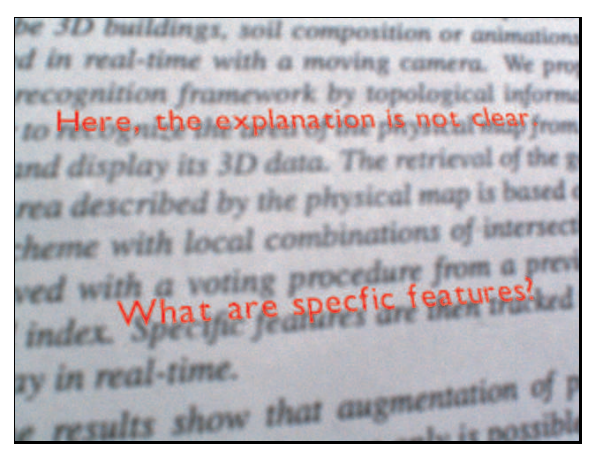

(c)

Fig. 2 Target objects. (a) An example of intersection maps. (b) 3D building visualization on the intersection map. (c) Virtual annotation on a document. 
4.2 Initialization

In our applications, a camera pose with respect to a $2 \mathrm{D}$ printed paper (map or document) is tracked. Before pose tracking, an initial pose is estimated as an initialization. Because the initialization needs an initial hash table (descriptor database) and keypoint database, these databases are prepared as follows.

To create an intersection map, the $2 \mathrm{D}$ distribution of intersections is computed and exported from a GIS. The distribution is equivalent to the top view of a map. By dealing with intersections as keypoints, the initial databases are created. For a text document, a document image is prepared from a digital document, such as a PDF. The image is also regarded as the top view of the document. By extracting keypoints from the image, the initial databases are created.

Because the initial databases are equivalent to the databases from a top view, a camera needs to be set at the top view for the initialization.

The process is the same as that of document image retrieval by (Nakai et al, 2006). In the initialization, keypoints are extracted from a captured image (keypoint extraction), and their indices (descriptors) are computed (descriptor computation). For each keypoint, a histogram of keypoint IDs is generated by retrieving keypoint IDs from the indices in the initial hash table. By selecting a peak of the histogram, each keypoint in the captured image has a corresponding keypoint in the keypoint database. In order to estimate a camera pose, a homography is computed from the correspondences, because the paper is set on a plane. Because there are outliers in the correspondences, we use RANSAC (Fischler and Bolles, 1981) to remove outliers and compute a refined homography.

\subsection{Online learning process}

A tilted camera pose cannot be computed from the initial hash table because keypoint matching by LLAH fails. In order to achieve wide base-line keypoint tracking, we propose online learning of descriptors.

The flowchart of pose tracking is illustrated in Figure 3. From keypoint extraction to pose estimation, the process is the same as the initialization. After pose estimation, the process moves to the descriptor update step. Because the camera pose is computed, keypoints in the keypoint database can be projected onto the image. By computing the distance between a projected keypoint and keypoints in the image, some of the keypoints in the image can get corresponding points in the keypoint database as matching by projection. For each keypoint for which correspondence is established, a descriptor update is performed.

\subsection{Neighbor keypoints' selection}

As described in Section 3, the neighbors of each keypoint are necessary in order to compute the descriptors. If we compute the distances for all keypoints from a keypoint, the computational cost is $O\left(N^{2}\right)$, where $N$ is the number of keypoints. The computation of all possible distances would imply large computational costs. To limit computational costs in the neighbor keypoints' selection, we limit the searching of candidates for distance computation to limited neighbor areas, in order to search neighbor keypoints efficiently.

As a pre-processing phase, the captured image is divided into square regions by segmenting them on a regular basis, as illustrated in Figure 4. When the keypoints are extracted 


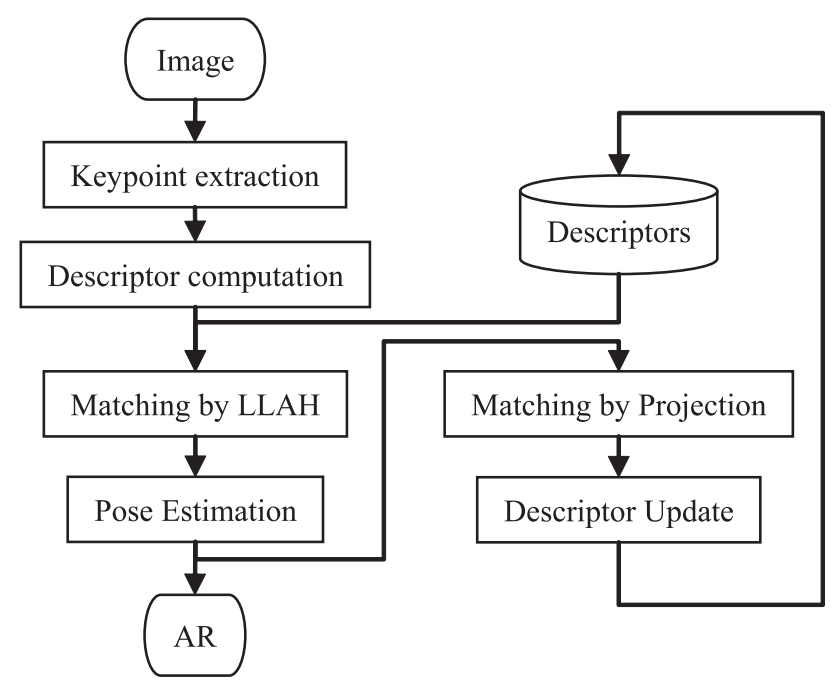

Fig. 3 Online learning process. As well as the initialization, a camera pose is estimated by matching by LLAH. After pose estimation, the keypoints in the keypoint database are projected onto the captured image to find correspondences in the image. If a correspondence is established, a descriptor update is performed.

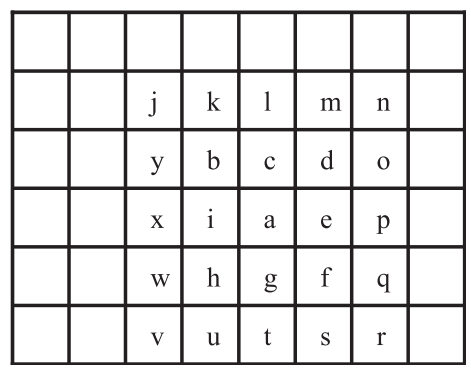

Fig. 4 Neighbor points selection. The image region is divided into square regions beforehand. If a keypoint is extracted in $\mathbf{a}$, candidates of neighbor points are collected from region a to region $\mathbf{i}$. If they are not sufficient, the candidates are collected from region $\mathbf{j}$ to region $\mathbf{y}$.

in the captured image, we compute the region to which each keypoint belongs. In addition, each region maintains the list of keypoints included in that region.

When we search the neighbor keypoints of a target keypoint, we collect potential candidates from the surrounding regions. For example, if a target keypoint belongs to a in Figure 4 , the candidates are extracted from region a to region $\mathbf{i}$. If the number of candidates is less than $n$ in Section 3, we collect more candidates from more surrounding regions. When the number is more than $n$, neighbor points are selected among the candidates by computing each distance. 
4.5 Matching by projection

In the pose estimation, we compute homography $\mathbf{H}$ as:

$$
\left[\begin{array}{c}
X \\
Y \\
1
\end{array}\right] \sim \mathbf{H}\left[\begin{array}{c}
x \\
y \\
1
\end{array}\right]
$$

where $(X, Y)$ is a keypoint in the keypoint database and $(x, y)$ is a keypoint in the image.

After the homography computation with RANSAC, we can obtain two types of outliers, as follows:

- Volatile keypoints by the instability of the keypoint extraction or motion blur.

- Keypoints stored in the keypoint database, but their descriptors are changed because of the narrow range invariance of the descriptors.

We ignore the first outliers because they are not useful for keypoint matching. For the latter outliers, a descriptor update is performed in order to keep these outliers as inliers.

When the homography is successfully computed, keypoints in the database can be projected onto the captured image by using inverse homography as

$$
\left[\begin{array}{c}
x^{\prime} \\
y^{\prime} \\
1
\end{array}\right] \sim \mathbf{H}^{-1}\left[\begin{array}{c}
X \\
Y \\
1
\end{array}\right]
$$

where $\left(x^{\prime}, y^{\prime}\right)$ is a projected keypoint from the database. For each outlier in the image $\mathbf{x}=$ $(x, y)$, we compute the distances between each projected keypoint $\mathbf{x}^{\prime}=\left(x^{\prime}, y^{\prime}\right)$ to find the nearest one. If the distance $\left|\mathbf{x}-\mathbf{x}^{\prime}\right|$ is less than a threshold (usually two pixels), the outlier is matched with the nearest projected keypoint to assign the keypoint ID of the projected keypoint.

\subsection{Descriptor update}

For all keypoints extracted from the image, ${ }_{n} C_{m}$ indices have already been computed in the descriptor computation. If a keypoint is an outlier, NULL is stored at some of the indices, because the indices (descriptors) were never computed. Because NULL means an empty index, we can update this index.

In Figure 5, some of the computed indices have NULL. For these indices, we fill NULL with a keypoint ID computed from matching by projection. The updated indices can be utilized after the next frame or later, so that outliers in the current frame become inliers in matching by LLAH. If there is a keypoint ID in the indices, the update is not performed.

The update is performed using a threshold for the number of the inliers after RANSAC based homography computation. If there are sufficient inliers in a frame, the update is not necessary for next frame. In Section 5.2, the influence of a threshold for the hash table is discussed.

Even though we insert keypoint IDs in the hash table, the size of the hash table does not change, because it has been pre-defined to a large size to access each index by $O(1)$. The preprocessing phase usually leads to many empty indices. In order to use those empty indices effectively, we insert keypoint IDs into the empty indices. In addition, the computational cost is not affected by the number of inserted indices because of the property of a hash scheme. 
This update helps the re-initialization of a camera pose when camera tracking fails. If there is no descriptor update, a camera should be set at the top view for the initialization, as described in Section 4.2. With the update, a camera pose can be re-initialized by setting back the camera on the camera trajectory.

Matching

\begin{tabular}{|c|c|}
\hline Index & ID \\
\hline$:$ & \\
\hline 14 & 423 \\
\hline$:$ & \\
\hline 164 & NULL \\
\hline$:$ & \\
\hline 345 & NULL \\
\hline$:$ & \\
\hline
\end{tabular}

Updated

\begin{tabular}{|c|c|}
\hline Index & ID \\
\hline$:$ & \\
\hline 14 & 423 \\
\hline$:$ & \\
\hline 164 & 311 \\
\hline$:$ & \\
\hline 345 & 311 \\
\hline$:$ & \\
\hline
\end{tabular}

Fig. 5 Update of LLAH. A keypoint has the indices 14, 164, and 345. If the keypoint gets 311 as a keypoint ID from matching by projection, the keypoint ID is inserted into 164 and 345. For 14, the update is not performed.

\subsection{Parallel processing}

In order to develop AR applications, we have to reduce computational costs as much as possible for real-time processing. We thus have to use computer resources effectively. Because we have two processing units in Intel Core 2 Duo architecture, it is important to assign the same load to each processing unit. To determiner the proper assignment, we first measure all processing times.

Average processing times were measured from an experiment by using 100 input images, as described in Table 1. In this case, the parameters for LLAH were as follows: $n=6$, $m=5$, and $l=4$.

The costs for keypoint extraction, descriptor computation and matching by descriptor are influenced by the number of keypoints captured in the image. Because the number of extracted keypoints usually varied between 500 and 600, the times in Table 1 are averages, but also include a variation range. When there are many outliers, the homography computation takes time, especially to get a refined homography. We have limited the number of iterations in RANSAC to 500, thus the maximum time was $22 \mathrm{~ms}$. When there are mostly inliers, this computation takes approximately $2 \mathrm{~ms}$.

Given those results, we have divided our process into two parts. The first thread takes care of "Capture an image" and "Keypoint extraction" while the other does the remaining tasks. 
For this parallel processing, we need two memory spaces to store keypoints. First, one memory space contains keypoints at the $t$ frame extracted by the first thread. The other memory space contains keypoints at the $t-1$ frame for the processes of the second thread. After all processes are finished in each thread, both threads are synchronized to copy the memory of the first thread to that of the second thread. If both threads work in the same way, the total cost per frame will be about $30 \mathrm{~ms}$.

Table 1 Processing time. We measured each processing time from 100 images. Processes are assigned to each thread depending on the result.

\begin{tabular}{cccc}
\hline Process & $\mathrm{ms}$ & Process & $\mathrm{ms}$ \\
\hline Image capture & 10 & $\begin{array}{c}\text { Homography computation } \\
\text { Keypoints extraction }\end{array}$ & $2(+20)$ \\
Matching by projection & 2 \\
Descriptor computation and matching by descriptor & $23 \pm 4$ & Descriptor update & 2 \\
\hline
\end{tabular}

\section{Experimental results}

5.1 Influence of LLAH parameters for keypoint matching

Because Nakai et al. only evaluated the accuracy of document image retrieval (Nakai et al, 2005, 2006), we evaluate the influence of LLAH parameters for keypoint matching. In this experiment, we prepared a white paper with 100 black circular dots randomly distributed to eliminate the influence of the instability in the keypoint extraction. The initial hash table and keypoint database are prepared from a top view image.

In the parameters of LLAH, we evaluate the influence of two parameters: $n$ and $m$, which mainly affect the computational costs and the accuracy. The other parameters were optimized to achieve the best result as $l=4, k=32$ and $H_{\text {size }}=2^{15}-1$. In this experiment, we tested the following combinations: $(n, m)=(5,5),(6,5),(7,5),(8,5)$ and $(7,6)$.

For each combination, we apply matching by LLAH to the same video capturing the paper from a top view to an inclined view around the center of the paper. We compute the angle between the vector from the center of a document to a camera position and the document plane using the tracking with the descriptor update at every frame as described in Section 5.2. The number of inliers after the RANSAC based homography computation is counted as illustrated in Figure 6.

First, we investigated the influence of $n$ as $(n, m)=(5,5),(6,5),(7,5)$ and $(8,5)$. In these cases, the descriptor dimension is ${ }_{5} C_{4}=5$. As illustrated in Figure $6, n=5$ got the least number of inliers. As $n$ increased from 6 to 8, the number of inliers increased because the number of descriptors for a keypoint increased. However, the computational cost is increased as ${ }_{5} C_{5}=1,{ }_{6} C_{5}=6,{ }_{7} C_{5}=21$ and ${ }_{8} C_{5}=56$ in matching by LLAH. Because this is a trade-off, we have to select parameters depending on computer resources and accuracy requirements.

Afterwards, we have examined the influence of the descriptor dimension. We compared these two combinations: $(n, m)=(7,5)$ and $(7,6)$ for which we have the following descriptor dimensions: ${ }_{5} C_{4}=5$ and ${ }_{6} C_{4}=15$. As illustrated in Figure 6, the result of $(n, m)=(7,6)$ was worse than that of $(n, m)=(7,5)$ because the discriminative power was too important. 


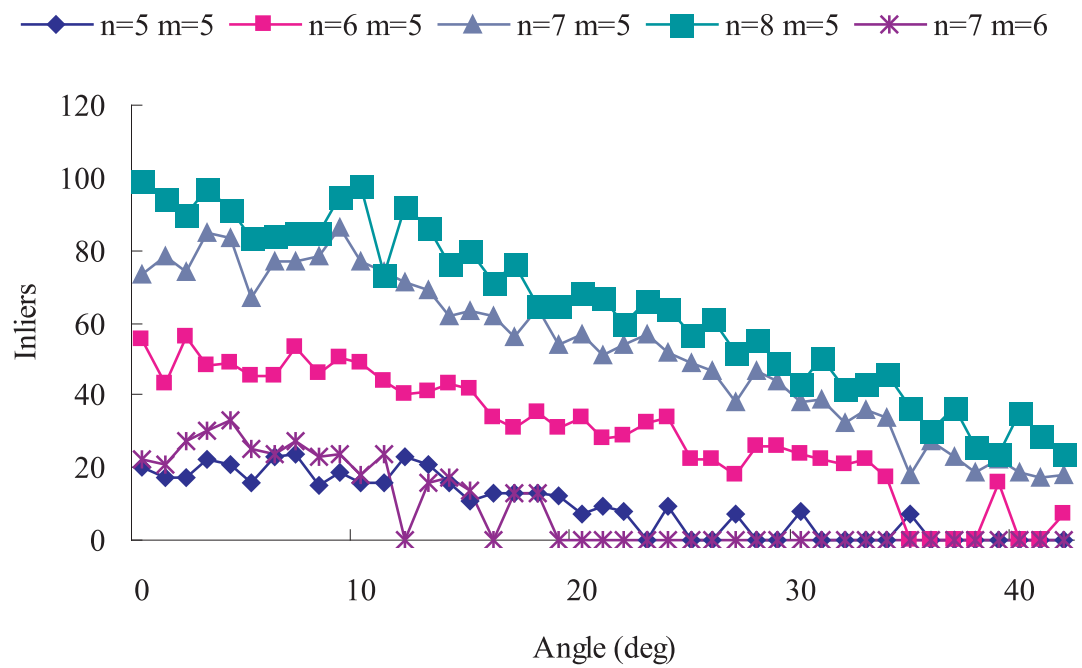

Fig. 6 The number of inliers after the RANSAC based homography computation. The combinations of $(n, m)=(5,5),(6,5),(7,5),(8,5)$ and $(7,6)$ are tested to check the relationship between inliers and angles.

\subsection{Behavior of descriptor update}

In this section, we investigate the behavior of the descriptor update for camera tracking. Because the descriptor update is performed depending on a threshold as described in Section 4.6, the influence of a threshold is investigated.

We tested those cases: no update, update when the number of inliers is less than 20, less than 40, and update at every frame. They are applied to the video utilized in Section 5.1. The LLAH parameters are as follows: $n=6, m=5, l=4, k=32$ and $H_{\text {size }}=2^{15}-1$.

Results are illustrated in Figure 7. The graph of "less than 20" is overlapped with the one with "no update" from 0 degrees to 27 degrees. Camera tracking does not fail with update at every frame and update when the number of inliers is less than 40. If the case of less than 20 inliers, the camera pose could not be estimated at some viewpoints. With update, the camera pose could be tracked up to 34 degrees. Compared to no update, the descriptor update allows camera tracking for a larger range of viewpoint changes.

Next, we investigated the behavior of the number of indices in the hash table as illustrated in Figure 8. The results naturally depend on the number of the descriptor update times, However, collisions in the hash table often happen as the number of updated indices increases. In an application, update of every frame may be acceptable when we use only one document. If we use many documents, it is important to effectively update descriptors in order to add descriptors in each document. As discussed in (Nakai et al, 2005), the appropriate parameters can be selected from several experiments and system environment. 
$\multimap$ No update $\longrightarrow$ Less than $20 \multimap-$ Less than $40 \multimap-$ At every frame

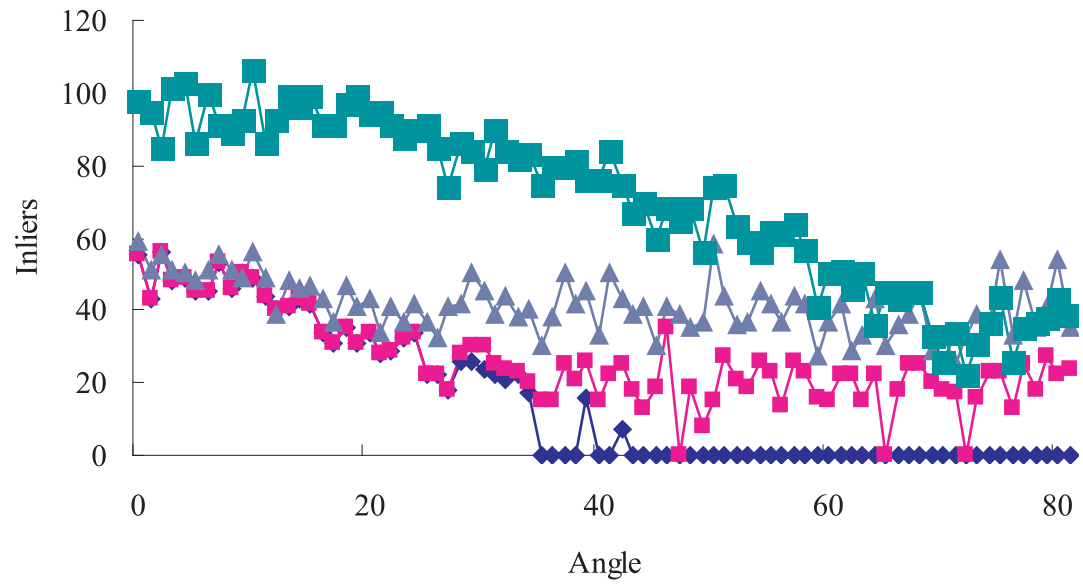

Fig. 7 Descriptor update with respect to a threshold. Whithout update, the camera could be tracked up to 34 degrees. In other cases, a camera pose is tracked.

$\longrightarrow$ Less than $20 \longrightarrow$ Less than $40 \longrightarrow$ At every frame

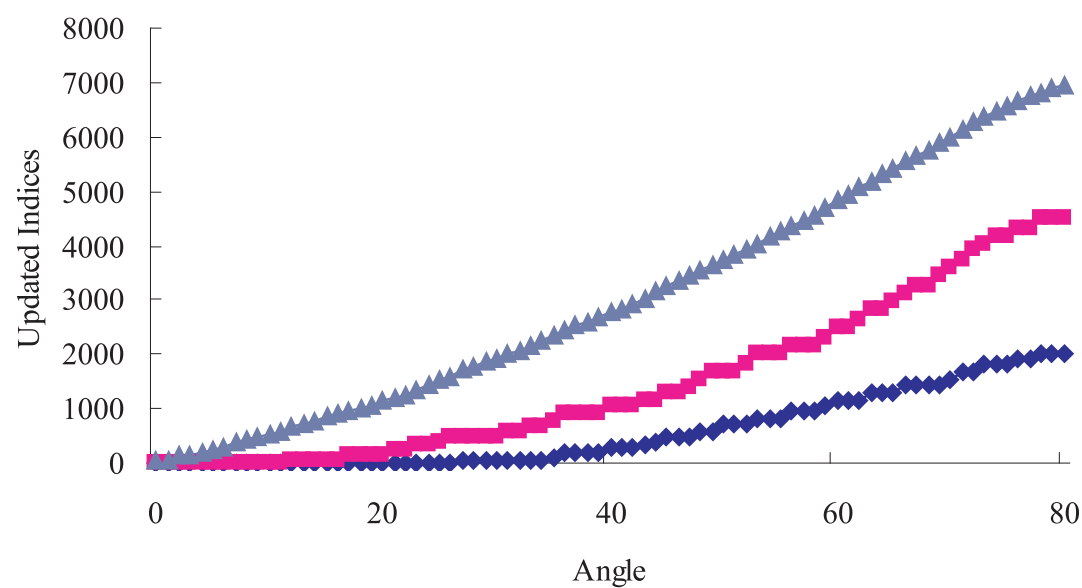

Fig. 8 Updated indices. The number of updated indices depends on the number of descriptor update times. 
5.3 Comparison with SURF

For intersection maps, local descriptors obviously do not work because local textures are the same and cannot be described to be distinct. In addition, these descriptors do not work well for text documents because of their repetitive patterns. To prove that the proposed tracking method is superior than these descriptors for documents, both tracking results on a document are compared. Because SIFT cannot run in real-time for AR systems, SURF OpenCV (?) implementation has been selected for the comparison.

For SURF tracking, a document image is prepared and printed on a A4 paper. The image resolution is selected as $672 \times 950$ because this made the best result compared to other resolutions. For each captured image, correspondences in the document image are established by the SURF descriptors, which can be regarded as matching between the top view and the captured image. In our tracking method, a document image is tracked by descriptor update at every frame. We applied both methods to a video capture from the top view to the inclined view around the center of a document. In each case, the number of inliers after RANSAC based homography computation is counted as illustrated in Figure 9.

For SURF, the matching failed after 32 degrees because SURF descriptors are not invariant to perspective distortion. In contrast, our tracking method succeeded at every frame and could estimate the angle of each captured image as shown in Figure 9. But, because our method is a framework for tracking by descriptor update, the descriptors in our tracking could be replaced by SURF descriptors.

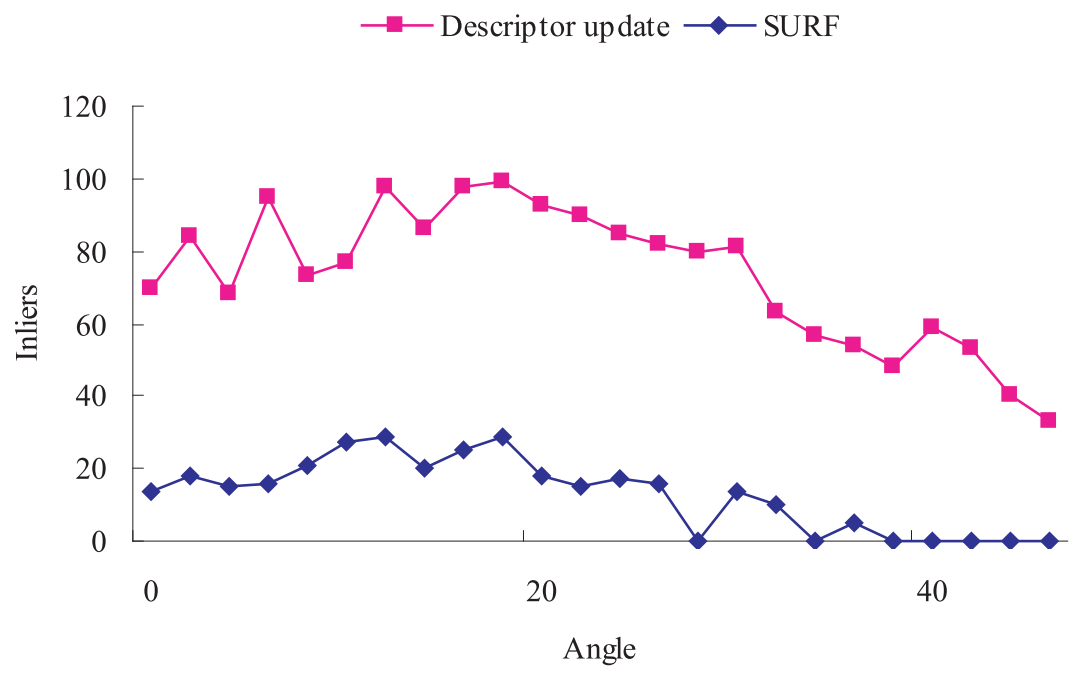

Fig. 9 Comparison with SURF. The number of inliers is compared in each method. Matching by SURF failed at 32 degrees. 


\section{Conclusions and future work}

We proposed a camera tracking method based on learning of the local arrangements of keypoints for intersection maps and text documents. To describe the arrangements, we use the LLAH that has already been used in studies of document image retrieval. Because the descriptors in LLAH are not invariant to a wide range of viewpoints, we proposed a dynamic learning process of the descriptors, called tracking by descriptor update.

In the updating process, the keypoints in the keypoint database are projected onto a captured image to establish the correspondences between the keypoints in the image and the projected keypoints as matching by projection. For each established correspondence, we insert the keypoint ID into the indices. From the experiment, the descriptor update contributes to a wide range of camera tracking.

In the future work, we have to efficiently handle a collision problem in a hash table. Because the purpose of the proposed method is to track a paper an intersection map or a document, the collision did not occur much yet.But if we handle multiple papers, collisions may happen many times, and the structure of the hash table will be a list at each index. In addition, we will develop natural keypoint matching by local arrangement of keypoints. The local arrangement may help the matching by local descriptors. The keypoint matching method will be utilized in various applications, such as SLAM.

Acknowledgements We thank Dr. Julien Pilet for the discussion. This work was supported in part by Grantin-Aid for JSPS Fellows and a Grant-in-Aid for the Global Center of Excellence for high-Level Global Cooperation for Leading-Edge Platform on Access Spaces from the Ministry of Education, Culture, Sport, Science, and Technology in Japan.

\section{References}

Arya S, Mount DM, Netanyahu NS, Silverman R, Wu A (1998) An optimal algorithm for approximate nearest neighbor searching fixed dimensions. J of the ACM 45:891-923

Bay H, Ess A, Tuytelaars T, Gool LV (2008) SURF: Speeded up robust features. CVIU 110:346-359

Datar M, Indyk P, Immorlica N, Mirrokni VS (2004) Locality-sensitive hashing scheme based on p-stable distributions. In: Proc. SCG, pp 253-262

Drummond T, Cipolla R (1999) Real-time tracking of complex structures with on-line camera calibration. In: Proc. BMVC, pp 574-583

Fiala M (2005) Artag, a fiducial marker system using digital techniques. In: Proc. CVPR, pp 590-596

Fischler MA, Bolles RC (1981) Random sample consensus: a paradigm for model fitting with applications to image analysis and automated cartography. C of the ACM pp 381395

Harris C, Stephens M (1988) A combined corner and edge detector. In: Proc. AVC, pp 147151

Hull J, Erol B, Graham J, Ke Q, Kishi H, Moraleda J, Van Olst D (2007) Paper-based augmented reality. In: Proc. ICAT, pp 205-209

Kato H, Billinghurst M (1999) Marker tracking and hmd calibration for a video-based augmented reality conferencing system. In: Proc. IWAR

Klein G, Murray D (2008) Improving the agility of keyframe-based SLAM. In: Proc. ECCV, pp 802-815 
Kotake D, Satoh K, Uchiyama S, Yamamoto H (2007) A fast initialization method for edgebased registration using an inclination constraint. In: Proc. ISMAR, pp 239-248

Lamdan Y, Wolfson H (1988) Geometric hashing: A general and efficient model-based recognition scheme. In: Proc. ICCV, pp 238-249

Lepetit V, Pilet J, Fua P (2004) Point matching as a classification problem for fast and robust object pose estimation. In: Proc. CVPR, pp 244-250

Lowe DG (2004) Distinctive image features from scale-invariant keypoints. IJCV 60:91-110

Mikolajczyk K, Schmid C (2005) A performance evaluation of local descriptors. PAMI 27:1615-1630

Nakai T, Kise K, Iwamura M (2005) Hashing with local combinations of feature points and its application to camera-based document image retrieval: Retrieval in 0.14 second from 10,000 pages. In: Proc. CBDAR, pp 87-94

Nakai T, Kise K, Iwata K (2006) Use of affine invariants in locally likely arrangement hashing for camera-based document image retrieval. In: Proc. DAS, pp 541-552

Nister D, Stewenius H (2006) Scalable recognition with a vocabulary tree. In: Proc. CVPR, pp 2161-2168

Pentenrieder K, Bade C, Doil F, Meier P (2007) Augmented reality-based factory planning - an application tailored to industrial needs. In: Proc. ISMAR, pp 1-9

Rosten E, Drummond T (2006) Machine learning for high speed corner detection. In: Proc. ECCV, pp 430-443

Sinha S, Frahm J, Pollefeys M, Genc Y (2006) GPU-based video feature tracking and matching. In: Proc. EDGE

Uchiyama H, Saito H (2009) Augmenting text document by on-line learning of local arrangement of keypoints. In: Proc. ISMAR, pp 95-98

Uchiyama H, Saito H, Servières M, Moreau G (2008) AR representation system for 3D GIS based on camera pose estimation using distribution of intersections. In: Proc. ICAT, pp 218-225

Uchiyama H, Saito H, Servières M, Moreau G (2009) AR GIS on a physical map based on map image retrieval using LLAH tracking. In: Proc. MVA, pp 382-385

Wagner D, Langlotz T, Schmalstieg D (2008a) Robust and unobtrusive marker tracking on mobile phones. In: Proc. ISMAR, pp 121-124

Wagner D, Reitmayr G, Mulloni A, Drummond T, Schmalstieg D (2008b) Pose tracking from natural features on mobile phones. In: Proc. ISMAR, pp 125-134 